\title{
LATS2 promoter hypermethylation and its effect on gene expression in human breast cancer
}

\author{
SAKI MATSUI, NAOFUMI KAGARA, CHIEKO MISHIMA, YASUTO NAOI, MASAFUMI SHIMODA, \\ ATSUSHI SHIMOMURA, KENZO SHIMAZU, SEUNG JIN KIM and SHINZABURO NOGUCHI
}

\begin{abstract}
Department of Breast and Endocrine Surgery, Osaka University Graduate School of Medicine, Suita, Osaka 565-0871, Japan
\end{abstract}
Received July 15, 2015; Accepted November 7, 2016

DOI: $10.3892 / 01.2017 .7535$

\begin{abstract}
Tumor-specific promoter hypermethylation of large tumor suppressor, homolog 2 (LATS2), a tumor suppressor gene, has been investigated using methylation-specific polymerase chain reaction (MSP) assays in different types of human cancer producing conflicting results. The aim of the present study was to evaluate the methylation status of the LATS2 promoter region using bisulfite sequencing with a next generation sequencer for breast cancer. In the 11 patients enrolled in the present study, the LATS2 promoter methylation index (MI) was uniformly high in tumor and normal tissues of the breast (median, 84.0 and $87.4 \%$, respectively). The presence of LATS2 promoter hypermethylation was confirmed in isolated tumor cells and normal epithelial cells using the magnetic-activated cell sorting method. In situ hybridization for LATS2 messenger RNA (mRNA) revealed that the mRNA expression of LATS2 was higher in normal epithelial cells, compared with tumor cells, however, it was not significantly associated with LATS2 MI. In 12 breast cancer cell (BCC) lines and two normal breast cell lines, the LATS2 promoter was uniformly hypermethylated with no correlation between the mRNA expression of LATS2 and the LATS2 MI. In addition, treatment of the BCC lines with a demethylating reagent had minimal effect on the mRNA expression of LATS2 in any of these cell lines. These results demonstrated that LATS2 hypermethylation was not involved in silencing the mRNA expression of LATS2 mRNA. The lower mRNA expression level of LATS2 in tumor cells, compared with normal epithelial cells, suggested the possible involvement of downregulation in the mRNA expression of LATS 2 in the pathogenesis of breast cancer. Therefore, the conflicting results previously reported for LATS2 promoter methylation in different types of cancer, detected using MSP assays may be attributable to the low fidelity of the MSP assay.
\end{abstract}

Correspondence to: Dr Naofumi Kagara, Department of Breast and Endocrine Surgery, Osaka University Graduate School of Medicine, 2-2-E10 Yamadaoka, Suita, Osaka 565-0871, Japan

E-mail: kagaran@onsurg.med.osaka-u.ac.jp

Key words: breast cancer, large tumor suppressor, homolog 2, epigenetics, methylation, expression

\section{Introduction}

Large tumor suppressor, homolog 2 (LATS2), is a tumor suppressor gene implicated in the regulation of cell proliferation, migration and apoptosis via several cell signaling pathways, particularly the Hippo, p53 and LATS-LIM domain kinase-actin pathways (1). Classically, a tumor suppressor gene is inactivated by a somatic mutation or promoter hypermethylation. Previous human cancer genome projects have revealed the presence of LATS2 mutations in several types of human cancer, including breast cancer, however, its frequency is low at $\sim 1 \%(2,3)$. Therefore, LATS2 mutations are unlikely to be important in the pathogenesis of the majority of breast cancer cases. By contrast, hypermethylation of the LATS2 promoter has been reported in a higher proportion of breast cancer (50\%) (4), acute lymphoblastic leukemia (ALL) (24\%) (5), astrocytoma (71.5\%) (6), lung cancer (78.8\%) (7), and nasopharyngeal cancer (36.7\%) (8) cases, and the absence of LATS2 hypermethylation has been confirmed in normal breast tissues (4) and normal brain tissues (6). In addition, the association between LATS2 promoter hypermethylation and reduced messenger RNA (mRNA) expression levels of LATS2 has been established in breast cancer (4), ALL (5) and astrocytoma (6). However, conflicting results have also been reported, namely, that LATS2 promoter hypermethylation is rare in head and neck cancer (9), and that it is more common in normal tissues, compared with tumor tissues in nasophalyngeal cancer (8) and gastric cancer (10).

Investigations of the methylation of promoter regions are usually performed through bisulfite modification to convert the methylation status into a sequence difference. The present study carefully examined the LATS2 promoter sequence following bisulfite modification and noted that several sequences with a high degree of homology to the LATS2 promoter sequence were present in intron 2 (pseudo promoter sequence). As previous methylation studies of the LATS2 promoter have used a methylation-specific polymerase chain reaction (MSP) assay, it is possible that the MSP assay may amplify the LATS2 promoter sequence and also the pseudo promoter sequences in intron 2, resulting in the conflicting results of LAST2 promoter methylation previously reported (4-10). This indicates that a more accurate technique is required for the investigation of LATS2 promoter methylation. Advancements in sequence technology with the development of next generation sequencing 
(NGS), has made it possible to quantitatively determine, with high accuracy, the methylation status of a gene. It is anticipated that bisulfite sequencing with NGS can be used to evaluate the methlyation status of the LATS 2 promoter more accurately than is possible using a conventional MSP assay.

The aim of the present study was, first, to evaluate the methylation status of the LATS2 promoter region using bisulfite sequencing with NGS, and second, to clarify the correlation between LATS2 promoter methylation and gene expression in breast cancer, using breast cancer cell (BCC) lines and breast cancer tissues.

\section{Materials and methods}

Patients and breast tumor samples. Pairs of tumor tissue and normal tissue samples were obtained from 11 patients with primary breast cancer, who underwent breast conserving surgery or mastectomy between 2001 and 2004 at Osaka University Hospital (Osaka, Japan). Patients treated with neoadjuvant chemotherapy and/or hormonal therapy were excluded. The clinicopathological characteristics of the patients recruited are summarized in Table I. The tissue samples were snap frozen in liquid nitrogen and stored at $-80^{\circ} \mathrm{C}$ until use. The remaining surgical specimens were fixed with $10 \%$ buffered formaldehyde. The present study was approved by the Institutional Review Board for Clinical Research of Osaka University Hospital, and informed consent was obtained from each patient prior to surgery.

DNA extraction and sodium bisulfite treatment. Total DNA from cell lines was isolated using TRIzol ${ }^{\circledR}$ reagent (Invitrogen; Thermo Fisher Scietnific, Inc., Waltham, MA, USA), and the DNeasy ${ }^{\circledR}$ Blood and Tissue kit (Qiagen, Inc., Valencia, CA, USA) was used to extract total DNA from the breast tissues. The genomic DNA $(1 \mu \mathrm{g})$ was treated with sodium bisulfite using an EpiTect ${ }^{\circledR}$ Bisulfite kit (Qiagen, Inc.).

Quantitative LATS2 promoter methylation analysis using NGS. The GS Junior system (Roche Diagnostics, Basel, Switzerland) was used for the NGS methylation assay for region $X$ (Fig. 1A) in accordance with the manufacturer's protocol, and data were analyzed using GS Amplicon Variant Analyzer software (version 2.7; Roche Diagnostics) (11). The LATS2 gene sequence was obtained from the University of California Santa Cruz browser (NM_014572, December 2013; GRCh38/hg38; https://genome.ucsc.edu). The MI was calculated by dividing the number of reads of cytosine by the total number of reads at each $\mathrm{CpG}$ site. Sequence alignment between regions $\mathrm{X}$ and Z1-4 of the LATS2 are shown in Fig. 1B. The NGS primers for region $X$, used for the frozen tissues and cell lines (NGS primer X-long; Fig. 1A), were as follows: Forward 5'-TTTTTTAGAAAGAAAATATATATGGGGAGG-3' and reverse 5'-AAA AAA ACCACCAAATTACCA ACTAAT AAC-3' (chr13: 21,061,921-21,062,405). For all 16 CpG sites, the average $\mathrm{MI}$ of $13 \mathrm{CpG}$ sites was defined as the LATS2 MI (2nd-14th CpG; Fig. 2A). The NGS primers for region X, used for the DNA extracted from formalin-fixed paraffin-embedded (FFPE) tissues (NGS primer X-short, Fig. 1A), were as follows: Forward 3'-TTATTGGGATAGTGGAATTAAATA ATTAAG-5' and reverse 3'-AAAATTTCTCCAAATTAA
Table I. Clinicopathological characteristics of patients/tumors for comparison of the large tumor suppressor, homolog 2 methylation in paired breast tumor and normal breast tissues.

\begin{tabular}{|c|c|}
\hline Characteristic & Patients, n (\%) \\
\hline \multicolumn{2}{|l|}{ Age (years) } \\
\hline$<50$ & $4(36)$ \\
\hline$\geq 50$ & $7(64)$ \\
\hline \multicolumn{2}{|l|}{ Menopausal status } \\
\hline Premenopausal & $4(36)$ \\
\hline Postmenopausal & $7(64)$ \\
\hline \multicolumn{2}{|l|}{ Tumor size $(\mathrm{cm})$} \\
\hline$\leq 2$ & $3(27)$ \\
\hline$>2$ & $8(73)$ \\
\hline \multicolumn{2}{|l|}{ Lymph node metastasis } \\
\hline Negative & $8(73)$ \\
\hline Positive & $3(27)$ \\
\hline \multicolumn{2}{|l|}{ Histological type } \\
\hline Invasive ductal carcinoma & $11(100)$ \\
\hline Other & $0(0)$ \\
\hline \multicolumn{2}{|l|}{ Histological grade } \\
\hline 1 and 2 & $5(45)$ \\
\hline 3 & $6(55)$ \\
\hline \multicolumn{2}{|l|}{ Estrogen receptor } \\
\hline Negative & $6(45)$ \\
\hline Positive & $5(55)$ \\
\hline \multicolumn{2}{|l|}{ Progesterone receptor } \\
\hline Negative & $6(45)$ \\
\hline Positive & $5(55)$ \\
\hline \multicolumn{2}{|c|}{ Human epidermal growth factor receptor 2} \\
\hline Negative & $7(64)$ \\
\hline Positive & $4(36)$ \\
\hline
\end{tabular}

TCAAACTAATCT-5' (chr13: 21,061,987-21,062,136). The NGS short primer included six $\mathrm{CpG}$ sites corresponding to the 10-15th $\mathrm{CpG}$, and the average $\mathrm{MI}$ of five $\mathrm{CpG}$ sites (10-14th CpG) was defined as the LATS2 MI. The NGS primers for region Y (NGS primer Y; Fig. 1A) were as follows: Forward 5'-GGGTAAATATTTAAGTTTGGGGGTA-3' and reverse 5'-AACCCCCTCACCTCCAAAAAC-3' (chr13: 21061335-21061816), and the NGS primers for region Z1 (NGS primer Z1, Fig. 1A) were as follows: Forward 5'-TTATGTTTGGTTAGAAATTTTTTTT-3' and reverse 5'-ATCTACCAACACAATACCCAATACATA-3 (chr13: 21,039,732-21,040,116).

In situ hybridization (ISH) for LATS2 $m R N A$. The QuantiGene ${ }^{\circledR}$ ViewRNA ISH Tissue Assay kit (Affymetrix, Inc., Santa Clara, CA, USA) was used in accordance with the manufacturer's protocol. FFPE sections ( $4 \mu \mathrm{m}$ thick) of the tumor tissues were incubated for $10 \mathrm{~min}$ at $98^{\circ} \mathrm{C}$ with $200 \mathrm{ml}$ $1 \mathrm{X}$ pretreatment solution (Affymetrix, Inc.), followed by protease digestion for $10 \mathrm{~min}$. The LATS2 View RNA ${ }^{\mathrm{TM}}$ Probe set (Affymetrix, Inc.) was hybridized for $2 \mathrm{~h}$ at $40^{\circ} \mathrm{C}$ using 
A

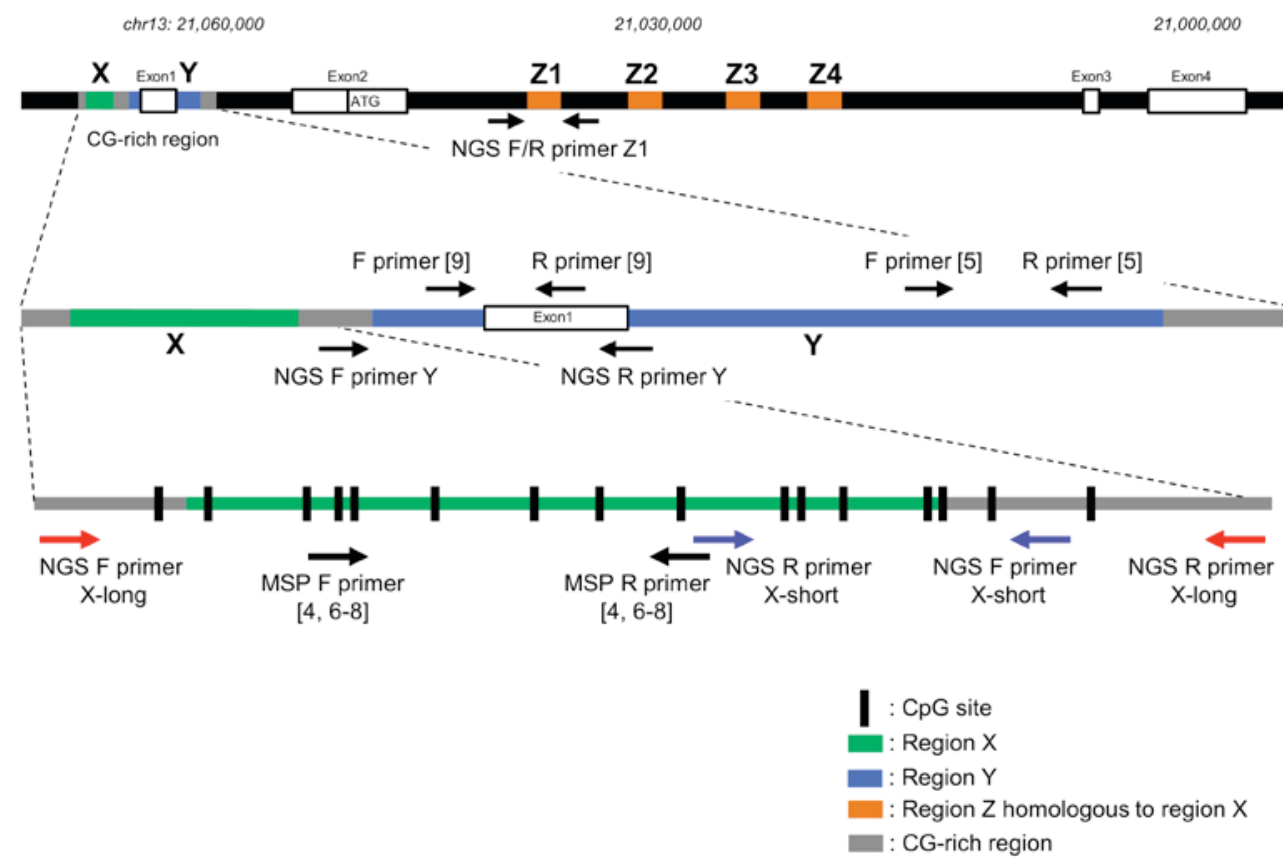

B

X UTTUTUAGAAAGAAATATAUATGGGGAGgggaggttgattaaat taaatuaggttaaatttgggtgtguatuagaugttuttttttt Z1 aagugatauguutguutuaguutuuuaaagtautgggattautggugtggguuauuatguutggtuagaaatttutttutttutttttt Z2 uutgauutuatgatuuauuuauuutgguttuuuaaagtgutgggattauaggugtgaguuautauguuuatuuuuaatatut $t \mathrm{t} t \mathrm{tt} t \mathrm{t}$ Z3 tutggggaaggtutgtaaautuuutgggggguuuttuuugaggatguutguttgttttuuaaaguuuuutgagttgtgttttgttttgt Z4 aagtggggaguuatttguatgaaaatgtgttuuutgttututagaaagtatguuaatgagaagauaaatgagtttagggutttttttt

*** **ak** $*$

X tttttttgagauggagttttgututtuttguutaggutggagtguaatgatgugatutuaggtuauuguaauutuuguutuuugggtt Z1 tt ttttttgagatggagtttugututtattguuuaggutggaatauaatggagutatutugguttautguaauututguuttuuggatt Z2 tuttttttgagauagagtgtugutut-gttguuuagguuauagtguagtggtguaatuttgguuuautguaauututguutuuugggtt Z3 tt tt tuttgagagggagtttuaututtgttguuuaggutggagtguaatggtgugatutugtutuuutgutauututguutuutgggtt Z4 tt tttttttagatggagtutuautuutgttauguaggutggggtguagtgguaugatutuagutuautgugaautuuauutuutgggtt

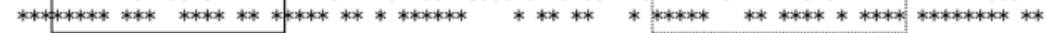

X uuagtgattututtguutuaguutuuugaagagutgggattauagguatgaguuauuauguutggutaa-ttttgtatttttaatagag Z1 uaagtgattutuutguutuaguutuuuaagtagutgggattauagguatguguuauuauguuuagutaa-ttttgtatttttagtagag Z2 uaagtgattutuutguutuagutttuugagtagutgggautauaggtgtguauuauuauauuuggutaatttttgtatttttagtagua Z3 uaagugattutuutguutuaguutuutgaatagutgaaattatagguatguguuauuatguuuagutaa-ttttgtatttttagtagag Z4 uaaguaattutuuttuutuaguutuuugagtagutgggat tauagguatguguuauuatguuuagutaa-ttttgtattttagtagag

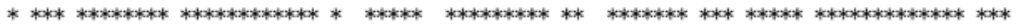

X auggGGGTTUTUUAAGTTGGTUAGGUTGGTUTugaautuuugauutuaggtgatuuauuug-uutagauututuaaagtgutgggatt Z1 atggga-tttuautatgttggtuaggttggtutugaautuutgauutuaggggatuuautuauuutuagut tuutaaagtgutgggatt Z2 atgggg-tttuauuatat tgguuaggutggtutugaautuutgauutu--gtgatauguutg-uuttgguutuuuaaagtuutgggatt Z3 augggg-tttutuuaggt tggtuaggutggtuttgaat tuuugauutuaggtgatuuaugug-gutuaguutuuuaaagtgutggaatt Z4 atgggg-ttttguuatgt tgguuaggutggtutugaautuutgauutuaggtgatuuauuug-uttugguutuuuaaagtgutaggatt

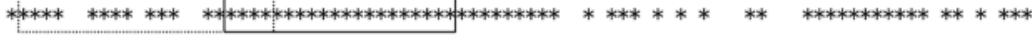

X auaggugtgaguuauuguttutgguaaaugtuuttUTTAATTGTTTGATTUUAUTGTUUUAATAGuugtutgtuuttttutguuuutat Z1 auaggtgtgagutguuttguutgguutuagaaatttatttutaauuuuaaggatgutuuatguattggguattgtgttgguagattgtu Z2 auagguatgaguuauuguauuugauat tuuaatatutttataaatguaatataaatuuaaauatuut tauuaaaatattagtaaatgg

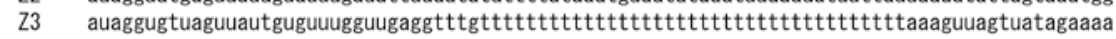
Z4 auagguatgaguuauuauguuuaguuguutttttttttttttttttttgagagattaaaaaaaaauaatuuagutggatguggtg

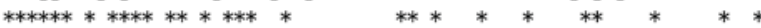

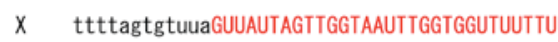

Z1 atgttuuugtuttgututuuutgtuttagguagtgtututgs

Z2 uatttggtaagaagatgatauatuaggatuaautgggatata

Z3 tgtgtuatttggtggauutuuutuuuatuuttttgaaatuut

Z4 gutuauauutataatuuuaguautttgggaggutgagguagg

Figure 1. Schematic representation of the LATS2 gene and primer design for DNA methylation analysis of LATS2. (A) Two primer sets were designed for DNA methylation analysis of LATS2 (region X) using NGS, including NGS primers for DNA from cell lines and frozen tissues (X-long; red arrows) and from formalin-fixed paraffin-embedded tissues (X-short; blue arrows). MSP primers used in previous studies (4-9), and NGS primers for regions Y and Z1 are also shown. The LATS2 genome sequence was obtained from the University of California Santa Cruz browser (NM_01,4572, December 2013; GRCh38/hg38). (B) Sequence alignment between regions X and Z1-4 of the LATS2 gene. Bisulfite converted cytosines are shown as ' $u$ '. Regions corresponding to X and Z1-4 are indicated with gray bars. The X-long and X-short NGS primers were designed for the unmatched region (red and blue capital letters, respectively). The locations of MSP primers and Sanger sequence primers used in previous studies $(4,6-8)$ are indicated by solid and dotted lines, respectively. LATS2, large tumor suppressor, homolog 2; NGS, next generation sequencing; MSP, methylation-specific polymerase chain reaction; F, forward; R, reverse. 
A

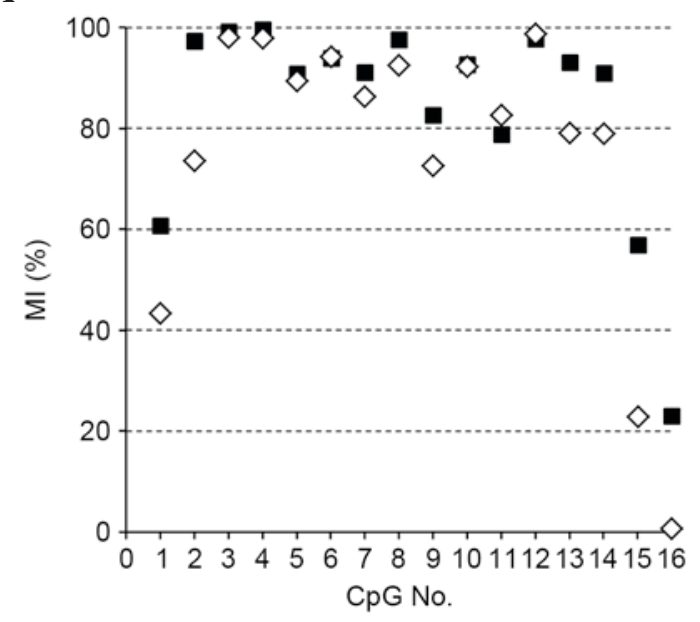

B

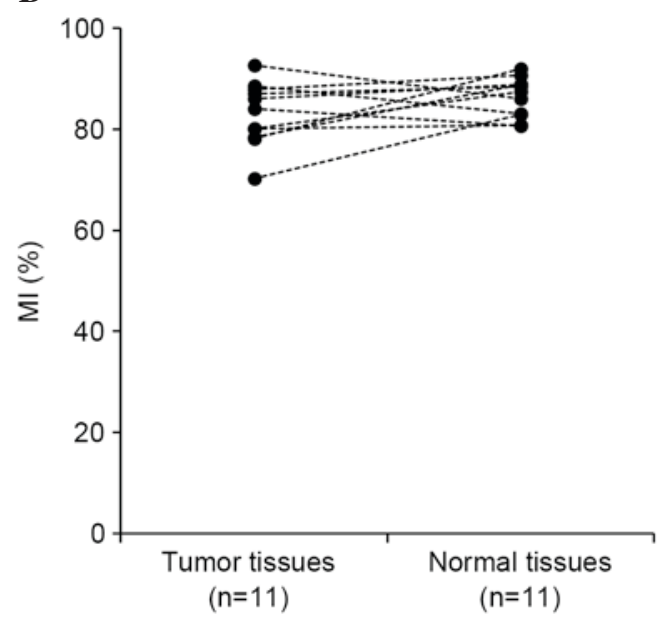

Figure 2. Methylation status of LATS2 in breast cancer and normal breast tissues. (A) Representative results for LATS2 MI in paired normal breast tissues (white diamonds) and cancer tissues (black squares). The average MI percentages of CpG sites 2-14 were used for statistical analyses. (B) Comparison of LATS2 MI in paired normal breast and cancer tissues. LATS2, large tumor suppressor, homolog 2; MI, methylation index.

a Hybridizer (Dako, Glostrup, Denmark). ISH images were captured using a fluorescent microscope (BX63; Olympus, Tokyo, Japan). Signal intensity was semi-quantitatively classified into four categories $(0$, negative; $1+$, weak; $2+$, intermediate; and 3+, strong) based on the number of cytoplasmic fluorescent dots in five non-overlapping fields at high-power magnification (x400).

Isolation of breast tumor cells using magnetic-activated cell sorting (MACS). The breast cancer cells and normal epithelial cells were isolated from the FFPE tissues using the MACS method with the EasySep Human EpCAM Positive Selection Cocktail, the EasySep Human MUC1 Positive Selection Cocktail and EasySep Magnetic Particles (Stemcell Technologies, Inc., Vancouver, BC, Canada), as previously described (12). Total DNA was extracted from these isolated cells using the QIAamp ${ }^{\circledR}$ DNA FFPE Tissue kit (Qiagen, Inc.).

Cell lines and analysis of demethylation using 5-aza-2'-deoxycytidine (5-aza). The 12 BCC lines (MCF7, ZR75-1, T47D, ZR75-30, MDA-MB-361, BT474, SKBR3, AU565, MDA-MB-453, MDA-MB-231, MDA-MB-468 and BT-20) and two normal breast epithelial cell lines (MCF10A and HMEC) were cultured according to the culture guides of the American Type Culture Collection (Manassas, VA, USA). For the investigation of demethylation, the cultured cells were seeded into a six-well plate at a density of $1.5 \times 10^{5}$ cells/well, and treated with $5 \mu \mathrm{mol} / 1$ 5-aza (Sigma-Aldrich; Merck Millipore) or with dimethylsulfoxide as a control for $72 \mathrm{~h}$ at $37^{\circ} \mathrm{C}$, with the medium replaced every $24 \mathrm{~h}$.

$R N A$ extraction and reverse transcription-quantitative polymerase chain reaction ( $R T-q P C R)$ analysis. Total RNA was isolated from the cell lines using TRIzol ${ }^{\circledR}$ reagent (Invitrogen; Thermo Fisher Scientific, Inc.), and $1 \mu \mathrm{g}$ of total RNA was reverse-transcribed into single strand cDNA using random primers and the ReverTra Ace ${ }^{\circledR}$ qPCR RT kit (Toyobo Co., Ltd., Osaka, Japan). LATS2 and GAPDH TaqMan ${ }^{\circledR}$ Gene Expression Assays (Hs00324396_m1 and Hs02758991_g1; catalog nos.,
4351372 and 4331182, respectively; Applied Biosystems; Thermo Fisher Scientific, Inc.) were used for qPCR on the LightCycler 480 Real-time PCR System (Roche Diagnostics). The qPCR primers and probes used for the measurement of LATS 2 and GAPDH mRNA expression were designed by the Applied Biosystems (Thermo Fisher Scientific, Inc.). The cycling conditions were as follows: $95^{\circ} \mathrm{C}$ for $10 \mathrm{~min}$, followed by 40 cycles at $95^{\circ} \mathrm{C}$ for $15 \mathrm{sec}, 60^{\circ} \mathrm{C}$ for $60 \mathrm{sec}$ and $50^{\circ} \mathrm{C}$ for $10 \mathrm{sec}$. Absolute mRNA expression levels of LATS2 and GAPDH were determined by the standard amplification curves obtained by serially diluted PCR products with known concentrations. The expression of LATS2 was normalized to that of GAPDH, and each assay was performed in duplicate. The result for each of the 5-aza treated BCC lines was normalized with that of its untreated control.

Statistical analysis. The JMP statistical software package (version 10; SAS Institute, Cary, NC, USA) was used for statistical analyses. The association between the expression of LATS2 and MI was assessed using the Kruskal-Wallis test. The Wilcoxon signed-rank test was used for comparison of the expression levels of LATS2 in the cancer and normal tissues. All statistical analyses were two-sided and $\mathrm{P}<0.05$ was considered to indicate a statistically significant difference.

\section{Results}

Methylation status of the LATS2 promoter in breast tumor and normal tissues. In the present study, 11 pairs of frozen breast tumor and normal tissues were analyzed using an NGS-based methylation assay which involved bisulfite sequencing with NGS. Representative results for the LATS2 MI at each $\mathrm{CpG}$ site of region $\mathrm{X}$ are shown in Fig. 2A, demonstrating that its methylation was uniformly high in the tumor and normal tissues (median 84.0 and $87.4 \%$, respectively; Fig. 2B). The tumor cells and normal epithelial cells were then isolated using the MACS method from five pairs of FFPE tumor and normal tissues, respectively, and subjected to the NGS-based methylation assay. The isolated tumor cells exhibited high MI 
A

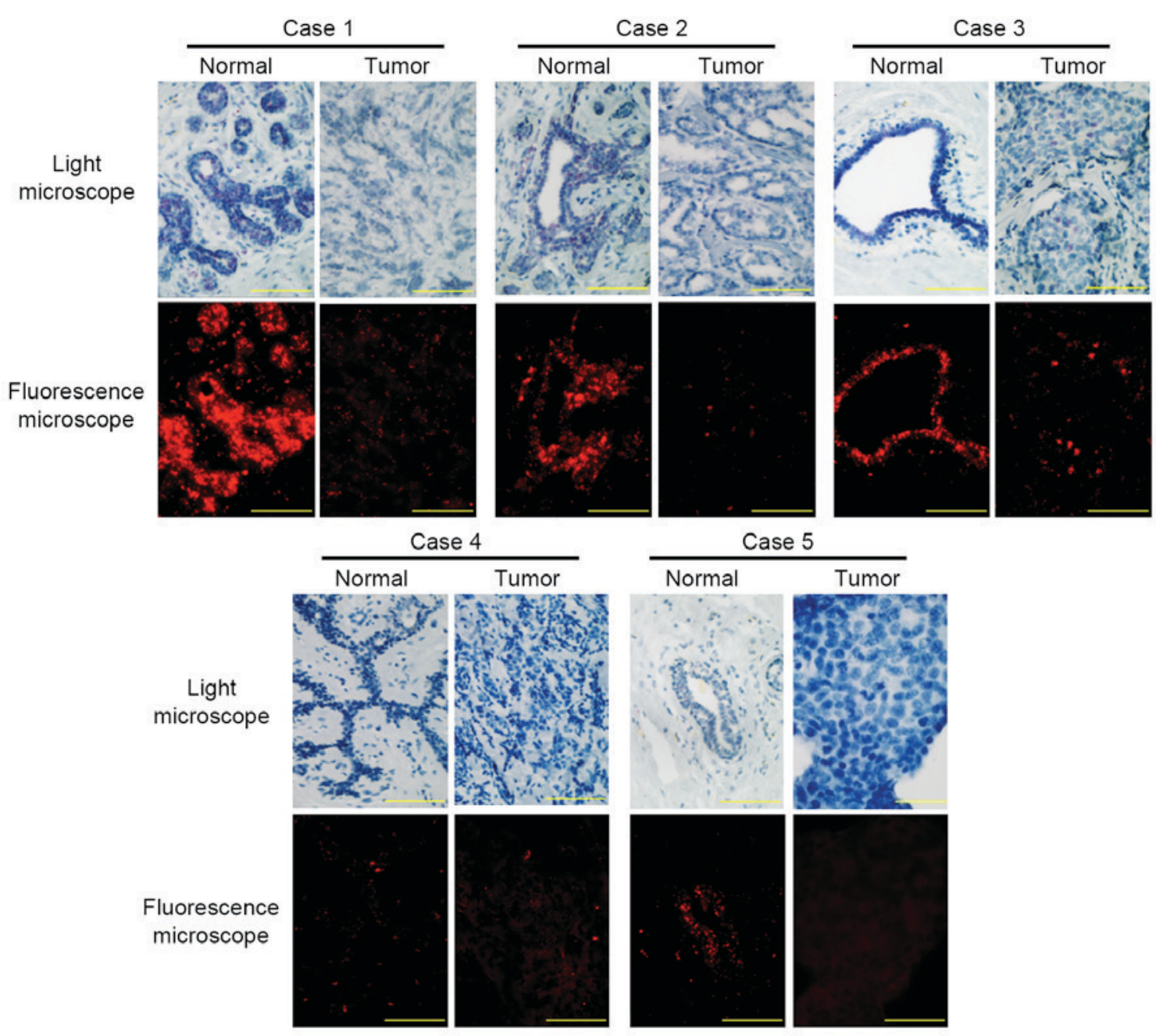

B

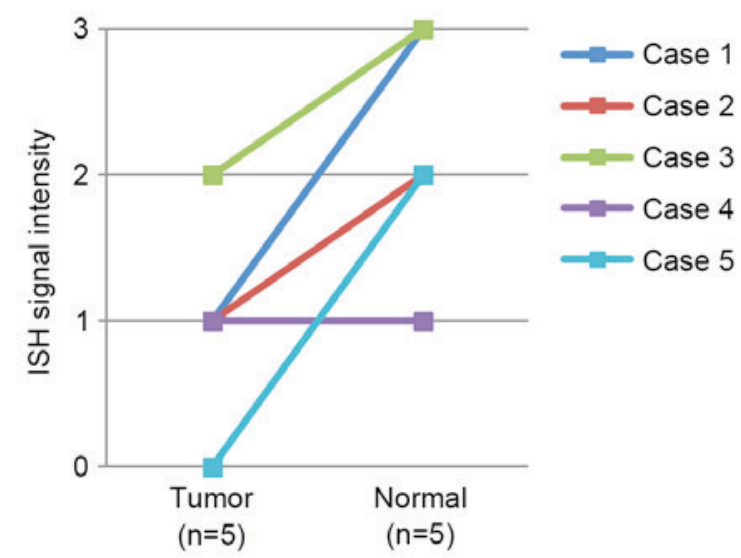

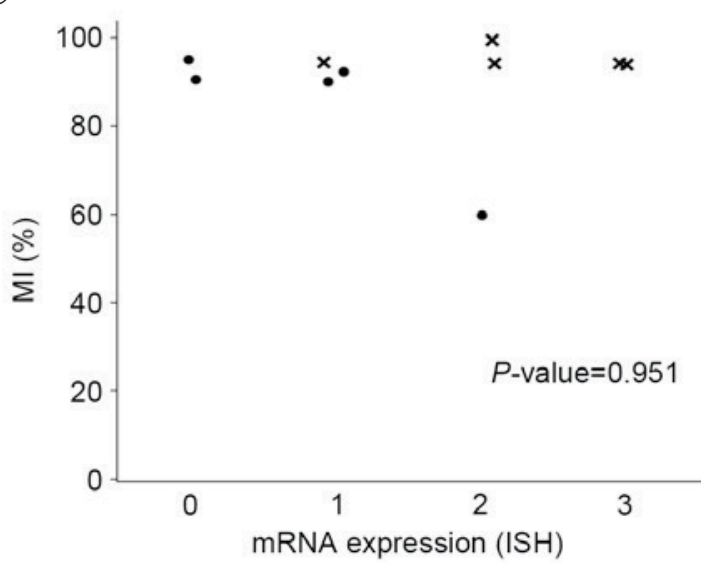

Figure 3. Representative results of ISH analysis of the mRNA expression of LATS2 in breast cancer tissues. (A) Results of ISH analysis of the mRNA expression of LATS2 in five paired breast cancer and normal tissues. Microscopic images were captured under a light microscope and a fluorescent microscope (magnification, $\mathrm{x} 400$; scale bars $=100 \mu \mathrm{m}$ ). (B) Comparison of ISH signal intensity in paired normal breast and cancer tissues. (C) Correlation between the mRNA expression of LATS2 and MI in normal breast tissue (x) and cancer tissues (black circles). ISH, in situ hybridization; LATS2, large tumor suppressor, homolog 2; MI, methylation index.

(median, 90.6\%; range, 59.9-95.1\%), as did the normal epithelial cells (median, 94.4\%; range, 94.1-99.7\%).

mRNA expression of LATS2 and its correlation with LATS2 promoter methylation in breast tumor and normal tissues.
ISH for LATS2 mRNA was used to examine the five pairs of FFPE breast tumor and normal tissues (Fig. 3A). In four of the five pairs, the mRNA expression of LATS2 was higher in the normal breast epithelial cells, compared with the tumor cells (Fig. 3B). No significant correlation was observed between 
A

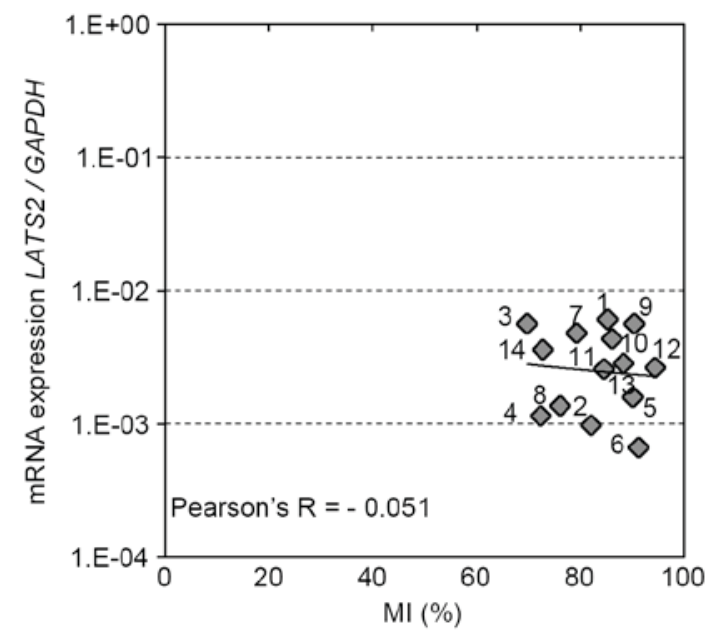

B

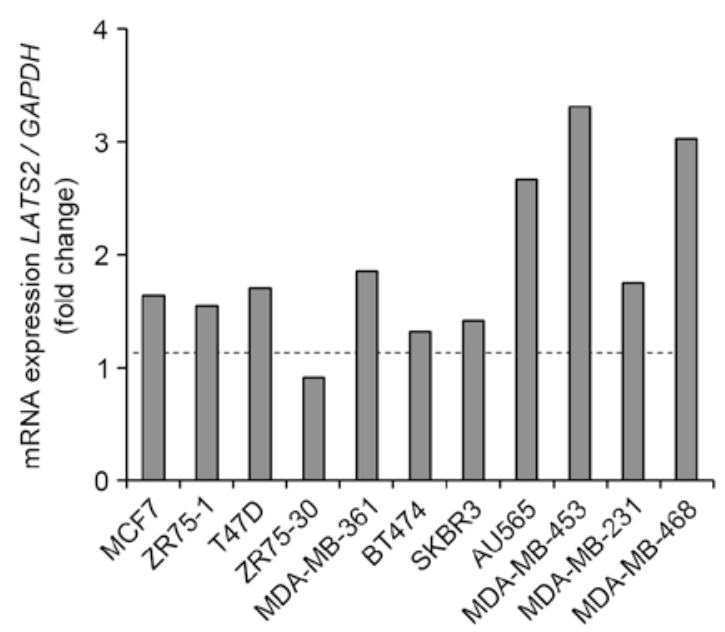

Figure 4. LATS2 methylation status of region X, the mRNA expression of LATS2 in breast cancer cell lines and the effect of demethylating reagent on mRNA expression. (A) Correlation between LATS2 MI and mRNA expression. The MI of LATS2 (region X) was determined using a next generation sequencing-based methylation assay and was compared with the mRNA expression of LATS2 in 12 BCC lines and two normal breast epithelial cell lines (MCF10A and HMEC). (B) Effect of 5-aza treatment on the mRNA expression of LATS2. The mRNA expression of LATS2 was evaluated following treatment with 5-aza or vehicle (dimethyl sulfoxide) in 11 BCC lines. The fold changes in the mRNA expression of LATS2 following 5-aza treatment are shown. LATS2, large tumor suppressor, homolog 2; BCC, breast cancer cell; MI, methylation index; 5-aza, 5-aza-2'-deoxycytidine; 1, MCF7; 2, ZR75-1; 3, T47D; 4, ZR75-30; 5, MDA-MB-361; 6, BT474; 7, SKBR3; 8, AU565; 9, MDA-MB-453; 10, MDA-MB-231; 11, MDA-MB-468; 12, BT-20; 13, MCF10A; 14, HMEC.

the mRNA expression of LATS2 and the LATS2 MI ( $\mathrm{P}=0.951$; Fig. 3C).

Promoter methylation of LATS2 and its effect on gene expression in BCC lines. To examine the methylation status of LATS2 region $\mathrm{X}$ in $\mathrm{BCC}$ lines, NGS-based methylation assays were performed using $12 \mathrm{BCC}$ lines and two normal breast cell lines. Similar to the findings for the breast tumor and normal tissues, the LATS2 promoter was found to be uniformly hypermethylated in all BCC lines and HMEC cells (Fig. 4A). Subsequently, the correlation between LATS2 promoter methylation and the mRNA expression of LATS2 was investigated using RT-qPCR analysis. No inverse correlation was observed between the mRNA expression of LATS2 and LATS2 MI (Pearson's correlation coefficient -0.051 ; Fig. 4A). Treatment of these BCC lines with a demethylating reagent ( $5 \mu \mathrm{M} 5$-aza) had minimal effect on the mRNA expression of LATS2 in any of the cell lines, suggesting that the mRNA expression of LATS2 was not directly regulated by methylation of its promoter (Fig. 4B). NGS-based methylation assays using eight BCC lines and two normal breast cell lines were then performed for region $\mathrm{Y}$ in the $\mathrm{CG}$-rich region located 333 bp downstream of region $\mathrm{X}$, and for region $\mathrm{Z1}$, which was most homologous to region $\mathrm{X}$ in intron 2 (Fig. 1A). Region Y was completely unmethylated and region $\mathrm{Z} 1$ showed a high level of methylation in all the cell lines. Neither the methylation status of regions $\mathrm{Y}$ or $\mathrm{Z1}$ was correlated with the mRNA expression of LATS2 (Fig. 5A and B).

\section{Discussion}

The present study investigated LATS 2 promoter methylation (region X; Fig. 1A) using NGS-based methylation assays, a more accurate method, compared with MSP assays. It was found that the LATS2 promoter exhibited a high level of methylation in breast tumor cells and normal breast epithelial cells, and that the mRNA expression of LATS2, determined using ISH, did not correlate with LATS2 promoter methylation. In addition, the LATS2 promoter exhibited high levels of methylation in all 12 BCC lines, regardless of their subtypes, and in two normal breast cell lines. Furthermore, it was established that treatment of BCC lines with a demethylating reagent had minimal effect on reactivation of the mRNA expression of LATS2. Taken together, these results demonstrated that LATS2 promoter hypermethylation was not implicated in the silencing of LATS2 in breast cancer and is unlikely to be important in the pathogenesis of breast cancer.

The present study showed that the mRNA expression of LATS2, determined using ISH, was lower in tumor cells, compared with normal breast epithelial cells, suggesting the possible involvement of the downregulation of LATS 2 mRNA in the pathogenesis of breast cancer. Such a reduction in the mRNA expression of LATS2 is likely to be induced by mechanisms other than promoter hypermethylation. Previously, several micoRNAs, including microRNA (miR)93 in breast cancer (13), miR25 in ovarian cancer (14) and miR31 in lung (15) and endometrial cancer (16), have been reported to directly target the LATS 2 gene and reduce its anti-oncogenic effect. A previous study identified a LATS2 mutation, which can reduce the expression of the gene in lung cancer, however, such a mutation in breast cancer remains to be elucidated $(2,3)$. Furthermore, the mechanism underlying the downregulated mRNA expression of LATS2 in tumor cells requires more detailed investigation.

Until now, reports regarding LATS2 promoter hypermethylation have been inconsistent in their support for tumor-specific LATS2 hypermethylation in various types of human cancer (4-9; Table II). Such inconsistency is most likely attributable to the low fidelity of the MSP assay used in these previous studies. A schematic representation of the LATS2 genome is shown in 
Table II. Summary of previous studies examining LATS2 methylation in human cancer.

\begin{tabular}{|c|c|c|c|c|c|}
\hline Author (year) & Type of Cancer & Tissue & $\mathrm{n}$ & $\begin{array}{c}\text { LATS2 methylation } \\
\text { positive rate }(\%)^{\mathrm{a}}\end{array}$ & Refs. \\
\hline \multirow[t]{2}{*}{ Visser and Yang (2010) } & \multirow[t]{2}{*}{ Breast } & $\mathrm{T}$ & 30 & 50 & \multirow[t]{2}{*}{ (1) } \\
\hline & & $\mathrm{N}$ & 6 & 0 & \\
\hline \multirow[t]{2}{*}{ Steinmann et al (2009) } & \multirow[t]{2}{*}{ Head and neck } & $\mathrm{T}$ & 54 & 80 & \multirow[t]{2}{*}{ (9) } \\
\hline & & $\mathrm{N}$ & 23 & 90 & \\
\hline \multirow[t]{2}{*}{ Zhang et al (2010) } & \multirow[t]{2}{*}{ Nasopharyngeal } & $\mathrm{T}$ & 30 & 37 & \multirow[t]{2}{*}{ (8) } \\
\hline & & $\mathrm{N}$ & 23 & 100 & \\
\hline \multirow[t]{2}{*}{ Jiang et al (2006) } & \multirow[t]{2}{*}{ Astrocytoma } & $\mathrm{T}$ & 88 & 72 & \multirow[t]{2}{*}{ (6) } \\
\hline & & $\mathrm{N}$ & 10 & 0 & \\
\hline \multirow[t]{2}{*}{ Sasakiet al (2010) } & \multirow[t]{2}{*}{ Lung } & $\mathrm{T}$ & 203 & 79 & \multirow[t]{2}{*}{ (7) } \\
\hline & & $\mathrm{N}$ & - & - & \\
\hline \multirow[t]{2}{*}{ Jiménez-Velasco et al (2005) } & \multirow[t]{2}{*}{ ALL } & $\mathrm{T}$ & 66 & 24 & \multirow[t]{2}{*}{$(5)$} \\
\hline & & $\mathrm{N}$ & - & - & \\
\hline
\end{tabular}

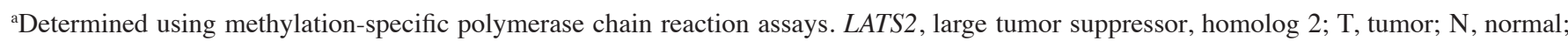
ALL, acute lymphoblastic leukemia.

A

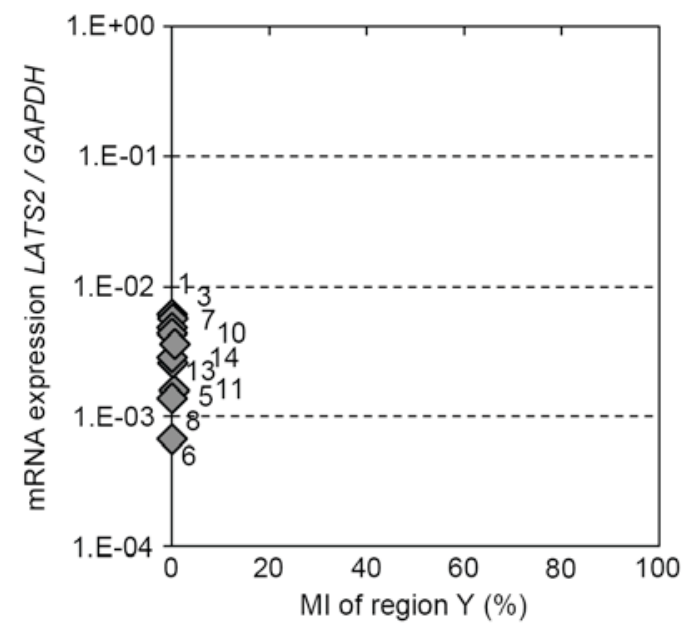

B

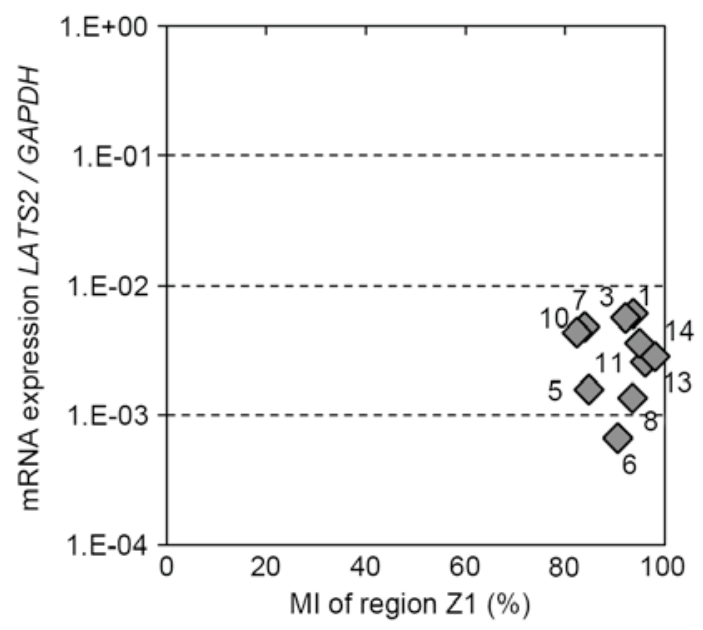

Figure 5. LATS2 methylation status of regions $\mathrm{Y}$ and Z1, and mRNA expression of LATS2 in breast cancer cell lines. Correlation between the LATS2 MI of (A) region Y or (B) region Z1 and mRNA expression. LATS2 MI was determined using a next generation sequencing-based methylation assay and was compared with the mRNA expression of LATS2 in eight breast cancer cell lines and two normal breast epithelial cell lines (MCF10A and HMEC). LATS2, large tumor suppressor, homolog 2; MI, methylation index; 1, MCF7; 3, T47D; 5, MDA-MB-361; 6, BT474; 7, SKBR3; 8, AU565; 10, MDA-MB-231; 11, MDA-MB-468; 13, MCF10A; 14, HMEC.

Fig. 1A. A careful survey of the LATS2 promoter sequence following bisulfite modification revealed the presence of several sequences (region Z1-Z4 in Fig. 1A) in intron 2, which exhibit a level of homology with the LATS2 promoter sequence. The most homologous sequence was region $\mathrm{Z1}$, which was $92 \%$ identical to region X (Fig. 1A), and located 22,024 bp downstream of region $X$. The alignment of the region $X$ and $\mathrm{Z} 1-\mathrm{Z} 4$ sequences following bisulfite modification is shown in Fig. 1B. The MSP primers used in our previous study (4) were designed on the basis of Sanger bisulfite sequencing. The primers used for bidirectional Sanger sequencing hybridize to regions $\mathrm{X}$ and $\mathrm{Z}$, and the sizes of the amplicons from the two regions were almost identical at $212 \mathrm{bp}$ in region $\mathrm{X}$ and 211 or $212 \mathrm{bp}$ in region $\mathrm{Z}$.
Therefore, Sanger sequencing may produce varied sequence results for regions $\mathrm{X}$ and $\mathrm{Z}$, leading to the faulty design of MSP primers (Table III). With such MSP primers, it is possible that the two regions are amplified at varying ratios depending on the PCR conditions, which raising concerns as to whether the assessment of LATS2 promoter methylation is accurate. By contrast, with NGS, accurate sequencing can be performed with a single base resolution, which avoids the possible misreading of similar sequences. In addition, the NGS primers were designed outside region $\mathrm{X}$ to avoid illegitimate hybridization (Fig. 1A). Therefore, the NGS-based methylation assay was considered sufficiently accurate to provide a true assessment of the methylation status of the LATS2 promoter. 
Table III. Primer sequences used in previous studies.

\begin{tabular}{lll}
\hline Author (year) & Primer & Concordance rate $(\%)$ \\
\hline
\end{tabular}

Sanger sequencing primers

Takahashi et al (2005)

\section{F 5'-TTTTGAGATGGAGTTTTGTT-3' \\ R 5'-AATTCAAAACCAACCTAACC-3'}

Methylation-specific primers

Takahashi et al (2005);

Jiang et al, (2006);

Sasaki et al, (2010);

Zhang et al, (2010)

Jiménez-Velasco et al (2005)

Steinmann et al (2009)

?

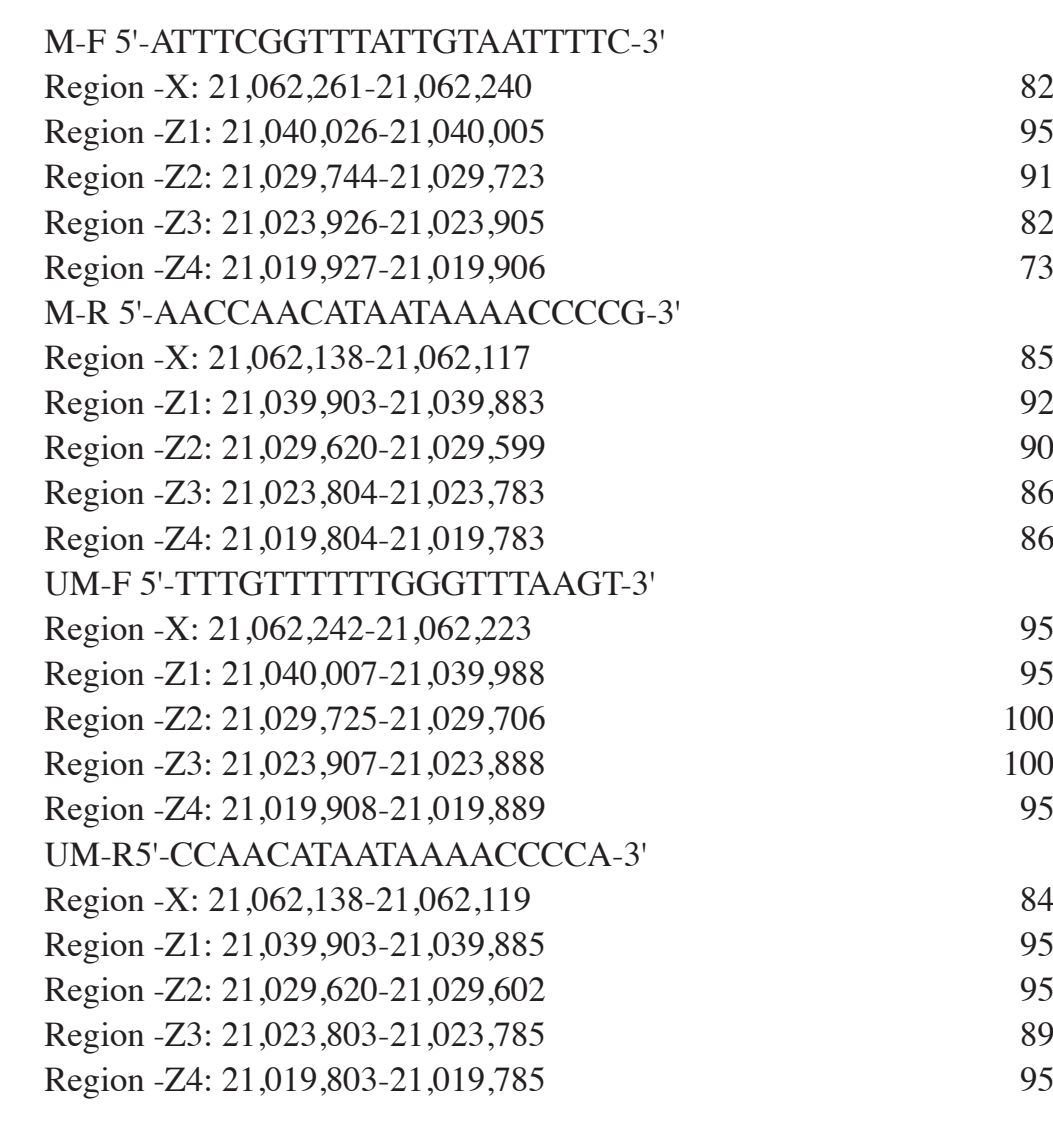

91

82

73

85

92

90

86

86

95

95

100

100

95

84

95

95

89
(4)

82

95

95

M-F 5'-GTTTTAGATTCGAAAGGTCGTAGC-3'

M-R 5'-AAAACTAATTAACCCGTAAAACGAT-3'

UM-F 5'-GGTGTTTTAGATTTGAAAGGTTGTAGT-3'

UM-R 5'-AAAAAACTAATTAACCCATAAAACAAT-3'

(9)

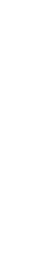

(1)

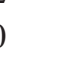

86

5


which contained the most $\mathrm{CpG}$ sites among regions Z1-4, in eight BCC lines and two normal breast cell lines, and found that the methylation status of region $\mathrm{Z} 1$ was uniformly high in all cell lines, and its methylation status was not associated with the mRNA expression of LATS2 (Fig. 5B). These results indicated that region $\mathrm{Z} 1$ was unlikely to be involved in the epigenetic regulation of the mRNA expression of LATS2.

In conclusion, the results obtained in the present study using NGS-based methylation assays demonstrated that LATS2 hypermethylation was not involved in the silencing of the mRNA expression of LATS2. The mRNA expression of LATS2 was lower in tumor cells, compared with normal epithelial cells, which suggested the possible involvement of the downregulation of LATS2 mRNA in the pathogenesis of breast cancer. However, the mechanism underlying this downregulation remains to be elucidated. The conflicting results previously reported for LATS2 promoter methylation obtained using MSP assays are considered to be attributable to the low fidelity of the MSP assay.

\section{References}

1. Visser S and Yang X: LATS tumor suppressor: A new governor of cellular homeostasis. Cell Cycle 9: 3892-3903, 2010.

2. Yu T, Bachman J and Lai ZC: Evidence for a tumor suppressor role for the large tumor suppressor genes LATS1 and LATS2 in human cancer. Genetics 195: 1193-1196, 2013.

3. Yu T, Bachman J and Lai ZC: Mutation analysis of large tumor suppressor genes LATS1 and LATS2 supports a tumor suppressor role in human cancer. Protein Cell 6: 6-11, 2015.

4. Takahashi Y, Miyoshi Y, Takahata C, Irahara N, Taguchi T, Tamaki Y and Noguchi S: Down-regulation of LATS1 and LATS2 mRNA expression by promoter hypermethylation and its association with biologically aggressive phenotype in human breast cancers. Clin Cancer Res 11: 1380-1385, 2005.

5. Jiménez-Velasco A, Román-Gómez J, Agirre X, Barrios M, Navarro G, Vázquez I, Prósper F, Torres A and Heiniger A: Downregulation of the large tumor suppressor 2 (LATS2/KPM) gene is associated with poor prognosis in acute lymphoblastic leukemia. Leukemia 19: 2347-2350, 2005.
6. Jiang Z, Li X, Hu J, Zhou W, Jiang Y, Li G and Lu D: Promoter hypermethylation-mediated down-regulation of LATS1 and LATS2 in human astrocytoma. Neurosci Res 56: 450-458, 2006.

7. Sasaki H, Hikosaka Y, Kawano O, Yano M and Fujii Y: Hypermethylation of the large tumor suppressor genes in Japanese lung cancer. Oncol Lett 1: 303-307, 2010.

8. Zhang Y, Hu CF, Chen J, Yan LX, Zeng YX and Shao JY: LATS2 is de-methylated and overexpressed in nasopharyngeal carcinoma and predicts poor prognosis. BMC Cancer 10: 538, 2010.

9. Steinmann K, Sandner A, Schagdarsurengin U and Dammann RH: Frequent promoter hypermethylation of tumor-related genes in head and neck squamous cell carcinoma. Oncol Rep 22: 1519-1526, 2009.

10. Lim B, Park JL, Kim HJ, Park YK, Kim JH, Sohn HA, Noh SM, Song KS, Kim WH, Kim YS and Kim SY: Integrative genomics analysis reveals the multilevel dysregulation and oncogenic characteristics of TEAD4 in gastric cancer. Carcinogenesis 35: 1020-1027, 2014.

11. Uji K, Naoi Y, Kagara N, Shimoda M, Shimomura A, Maruyama N, Shimazu K, Kim SJ and Noguchi S: Significance of TP53 mutations determined by next-generation 'deep' sequencing in prognosis of estrogen receptor-positive breast cancer. Cancer Lett 342: 19-26, 2014.

12. Otani Y, Miyake T, Kagara N, Shimoda M, Naoi Y, Maruyama N, Shimomura A, Shimazu K, Kim SJ and Noguchi S: BRCA1 promoter methylation of normal breast epithelial cells as a possible precursor for BRCA1-methylated breast cancer. Cancer Sci 105: 1369-1376, 2014.

13. Fang L, Du WW, Yang W, Rutnam ZJ, Peng C, Li H, O'Malley YQ, Askeland RW, Sugg S, Liu M, et al: MiR-93 enhances angiogenesis and metastasis by targeting LATS2. Cell Cycle 11: 4352-4365, 2012

14. Feng S, Pan W, Jin Y and Zheng J: MiR-25 promotes ovarian cancer proliferation and motility by targeting LATS2. Tumour Biol 35: 12339-12344, 2014.

15. Liu X, Sempere LF, Ouyang H, Memoli VA, Andrew AS, Luo Y, Demidenko E, Korc M, Shi W, Preis M, et al: MicroRNA-31 functions as an oncogenic microRNA in mouse and human lung cancer cells by repressing specific tumor suppressors. J Clin Invest 120: 1298-1309, 2010.

16. Mitamura T, Watari H, Wang L, Kanno H, Kitagawa M, Hassan MK, Kimura T, Tanino M, Nishihara H, Tanaka S and Sakuragi N: microRNA 31 functions as an endometrial cancer oncogene by suppressing Hippo tumor suppressor pathway. Mol Cancer 13: 97, 2014. 